$10-18-2011$

\title{
Depicting estimates using the intercept in meta- regression models: The moving constant technique
}

Blair T. Johnson Dr.

University of Connecticut - Storrs, blair.t.johnson@uconn.edu

Tania B. Huedo-Medina Dr.

University of Connecticut - Storrs, tania.huedo-medina@uconn.edu

Follow this and additional works at: https://opencommons.uconn.edu/chip_docs

Part of the Applied Statistics Commons, Biostatistics Commons, Clinical Trials Commons, Community Health and Preventive Medicine Commons, Educational Assessment, Evaluation, and Research Commons, Epidemiology Commons, Management Sciences and Quantitative Methods Commons, Multivariate Analysis Commons, Other Statistics and Probability Commons, Psychological Phenomena and Processes Commons, Statistical Methodology Commons, and the Statistical Models Commons

\section{Recommended Citation}

Johnson, Blair T. Dr. and Huedo-Medina, Tania B. Dr., "Depicting estimates using the intercept in meta-regression models: The moving constant technique" (2011). CHIP Documents. 33.

https://opencommons.uconn.edu/chip_docs/33 


\section{Depicting estimates using the intercept in meta-regression models: The moving constant technique}

Johnson, B. T., \& Huedo-Medina, T. B. ${ }^{\S}$ (2011). Depicting estimates using the intercept in meta-regression models: The moving constant technique. Research Synthesis Methods, 2(3), 204-220. (doi: 10.1002/jrsm.49)

\section{Blair T. Johnson ${ }^{a *}$ and Tania B. Huedo-Medina ${ }^{a}$}

In any scientific discipline, the ability to portray research patterns graphically often aids greatly in interpreting a phenomenon. In part to depict phencomena, the statistics and capabilities of meta-analytic models have grown increasingly sophisticated. Accordingly, this article details how to move the constan m weighted meta analysis regrsitios. Als ( $i z$. it is intercept) in such models can be indispensible when it is not relegated to its usual static role. The moving constant technique makes possible estimates and confidence intervals at moderator levels of interest as well as continuous confidence bands around the meta-regression line itself. Such estimates, in turn, can be highly informative to interpret the nature of the phenomenon being studied in the meta-analysis, especially when a comparison with an absolute or a practical criterion is the goal. Knowing the point at which effect size estimates reach statistical significance or other practical criteria of effect size magnitude can be quite important. Examples ranging from simple to complex models illustrate these principles.

Keywords: meta-regression; meta-analysis regression; weighted regression; point estimates; confidence intervals; confidence bands; prediction intervals; graphical displays

Across sciences, graphicaldisplays of iesuls can be an inmense statistical model tests (Light et al., 1994; Tufte, 2001). Meta-analysts have an increasingly rich array of options when it comes to displaying weighted mean effect sizes and individual effect sizes (Anzures-Cabrera \& Higgins, 2010; Borman and Grigg, 2009), showing how effect size indexes (Ts) such as the standardized mean difference (SMD) or logged odds ratio performs across a literature. Variations on such displays have also been used to imply how Ts may relate to an independent variable (Borman and Grigg, 2009, Lau et al., 2006), which meta-analysts as meta-regressions, where the weights are the inverse of the variance for each $T$. Analysts often plot $T s$ around the meta-regression line, which helps to capture the meaning of the meta-regression and to see how well it explains varibility in Ts (Borman and Grigg 2009; La et al 2006). Relatively rare to date are meta segression plots including confidence bands around the regression line to highlight at what levels of a moderator estimates exceed the null value or some benchmark criterion of clinical significance (Hayter et al 2007. Liu et al 2008). There also are few tools presently available to display patterns from meta-regressions with multiple moderator factors. With few exceptions, meta-analysts more often provide tabular and textual descriptions of their meta-regression results, providing standardized or unstandardized coefficients but not displaying how Ts vary at different points along the moderator variable or variables. Popular sources on meta-regression (e.g., Borenstein et al., 2009; Cooper et al., 2009; Hedges and Olkin, 1985; Higgins and Green, 2009; Lipsey and Wilson, 2001; Raudenbush, 2009) explain how this technique is applied and explicate the assumptions that underlie such analyses. They provide little detailed information on how one can extend these principles to produce confidence bands around the underlying regression line or confidence intervals at particular values of a moderator or moderators. By the same token, these reference works also do not address how to produce confidence intervals for estimates at particular values of interest along or

Department of Psychology, 406 Babbidge Road Unit 1020, University of Connecticut, Storrs, CT 06269-1020, USA: Center for Health Intenvention and Prevention 2006 Hillside Road Unit 1248, University of Connecticut, Storrs, CT 06269-1248, USA. *Correspondence to: Blair T.J.Johnson, Department of Psychology, 406 Babbidge Road Unit 1022, University of Connecticut, Storrs, CT 06269-1020, USA E-mail: blairts 
outside the observed range of the moderator variable. Treatises that have examined graphical displays also have left these issues unexplored (Borman and Grigg, 2009; Harbord and Higgins, 2008; Light et al., 1994; Viechtbauer, 2010a). Making matters worse, as we show, pre-packaged graphing software can badly mismatch conventiona meta-analytic assumptions when used for plotting confidence bands.

In this article, we illustrate how to move the constant in meta-regression models to plot confidence bands and to produce confidence intervals for $T s$ at particular values of the moderator variable. Our purpose is not to develop or elucidate the underlying statistical principles underlying conventional meta-regression modeling (for these, see, e.g., Higgins and Thompson, 2002; Konstantopoulos and Hedges, 2009; Raudenbush, 2009), defined as regressions weighted by the inverse of the variance for each $T$, but to show how fuller use of these principles can enrich guide the practice of regression with primanyel databases. We first consider guide the practice of regression with primary-level databases. We first consider estimates and confidence bands for constant technique in the case of multiplemoderator models, including nonlinear predicted values and an exploration of residuals. We conclude by discussing the limitations and potentials associated with such displays.

A bivariate example moving the constant

Meta-regression has become an extremely popular tool to see which moderators explain discrepancies in study findings, which is especially valuable in the face of heterogeneity, when the hypothesis of homogeneity has been rejected. That is, such Ts exhibit greater variability than sampling error alone would suggest. Meta-regression's popularity lies in its flexibility: It can include more than one predictor, which can help determine what moderator variables best explain unique variation in Ts (Borenstein et al., 2009; Hedges and Olkin, 1985). Meta-regressions can also incorporate both categorical and continuous moderator variables; the latter can be linear or nonlinear (e.g., quadratic, logarithmic, etc.). The fixed-effects version assumes that only sampling error is present among study findings whereas the mixed-effects version assumes that slopes are fixed but that the intercept is random, so between-study variance adds another source of error in the model (Hedges and Vevea, 1998). This latte approach is conventionally labeled random-effects meta-regression (Borenstein et al., 2009; Harbord and Higgins, fixed-effects (Berkey et al., 1995; Hedges, 1992; Huedo-Medina and Johnson, 2010; Knapp and Hartun, 2003; Viechtbauer, 2010a; Viechtbauer, 2010b). Each of these forms of meta-regression invokes weights that are the inverse of the variance for each $T$ (for calculation specifics, see Borenstein et al., 2009; Harbord and Higgins, 2008; Huedo-Medina and Johnson, 2010; Lipsey and Wilson, 2001).

The Moving Constant Technique. Like ordinary least squares regression models, meta-regression models include a single intercept and a slope for each moderator variable or covariate. Although it is commonly ignored in practice, the constant, or intercept, in a meta-regression equation can be extremely valuable because using it permits on to estimate confidence bands and intervals. Take as an example the meta-analysis of trials evaluating the success of antidepressants at alleviating depressive symptoms presented by Kirsch et al. (2008). They gathered Administration for drug approval. Each trial evaluated change in patients' depressive symptoms after a period taking antide ressants compared with those taking placebo; patients were randomly assigned to condition. Depression was assessed in each study with the Hamilton Rating Scale of Depression (HRSD). Kirsch and colleagues evaluated the hypothesis that antidepressants should succed better for more severely depressed samples of participants. They examined this hypothesis in two main ways. one focused on how much improvemed patients in the drug or placebo groups experienced over time and the other, on which we will focus in this example focused on the controlled comparison, the amount of improement if any, in drug relative to placebo at some point focter treatment commenced. $T$ was defined in terms of the arithmetic difference in the change of depression means between the two groups, as assessed using the HRSD. The improvement from baseline is obtained for each group, and positive values imply that the drug group improved more than the placebo group. Another purpose of their work was to evaluate for what levels of severity antidepressants achieve a clinically significant level of change. For this purpose, they adopted the 2004 recommendation of the UK's National Institute for Health and Clinical Excellence (NICE), which is three HRSD points better improvement for the drug group relative to the placebo group. Results confirmed the hypothesis presented by Kirsch and colleagues: antidepressant efficacy was indeed larger for samples with more extreme depression, Figure 1 (panel a) shows these findings; antidepressants efficacy did not reach a conventional value of clinical significance (green line) except in samples with very severe depression. Although such a display is useful for such purposes, a version with confidence bands would enable inferences not only about whether antidepressants have a statistically significant impact on depression (relative to placebo) but also, and more importantly, for samples with what levels of depression it has this effect. Unles otherwise noted, our demonstrations use pointwise confidence bands, which surround the meta-regression line, We addres two other alternatives, simultaneous confidence bands and prediction bands, in the final section.)
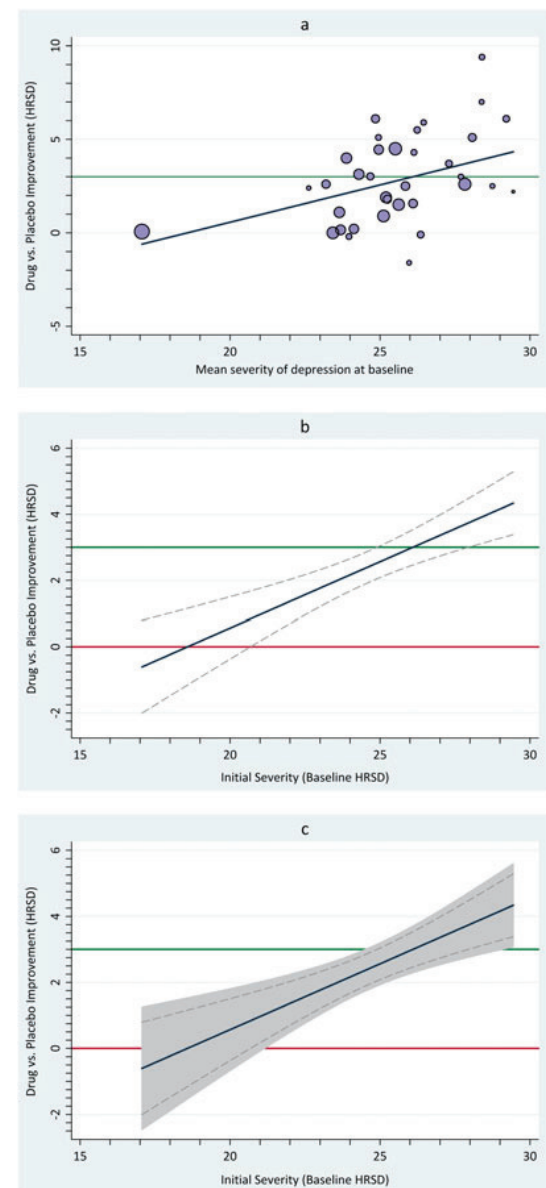
Figure 1. Improvement on depressive symptoms as a function of the samples' baseline severity of depression : (a) Kirsch et al's (2008) Figure 4 ,
reprinted with overall trends; (b) same trends but with confidence band addded; (C) same trends with confidence bands that follow differing
assumptions (see text for more description). HRSD, Hamilton Rating Scale of Depression

Imagine that instead we want to know whether antidepressants have a statistically significant benefit for groups with an average level of depression of 17 , the lowest value observed in any of the trials, which is in the "moderate" range of the scale. A starting point is the meta-regression equation of the line from their analysis:

$$
\hat{T}=-7.416+0.3991 \text { (Baseline depression), }
$$

where $\hat{T}$ is the estimated value of the difference between drug and placebo improvement change. By default, meta-regression statistical output includes a test of whether the coefficient for the slope differs significantly from 
Table 1. Meta-regression equations of the line in which the constant is moved to estimate the difference in Hamilton Rating Scale of Depression values for members of antidepressant groups relative to members of placebo groups for samples with varying levels of inita
Constant

Model With mean HRSD value

( $95 \%$ confidence

Width of confidence Slope $(95 \%$

\section{Original metric}

interval)

confidence interval

(constant $=0$ )

Original metric -17

Original metric -20

Original metric -23
Original metric -26

Original metric -29

Original metric -32
Original metric -46

$-7.416(-11.71,-3.12)$

8.59

$0.3991(0.23,0.57)$

$-0.630(-2.04,0.78)$

$0.567(-0.37,1.51)$

$1.765(1.21,2.32)$

$2.962(2.43,3.49)$

$.357(4.00,6.71)$

$10.940(7.23,14.66)$

2.82

$0.3991(0.23,0.57)$

$0.3991(0.23,0.57)$

$0.3991(0.23,0.57)$

$0.3991(0.23,0.57)$

$0.3991(0.23,0.57)$

$0.3991(0.23,0.57)$

Notes: The slope appears in this Table to help show that each model is estimating the same information. Rows that are rendered in italics are extrapolations beyond the observed data assumptions; estimates incorporating random-effects assumptions exhibited highly similar results (see Figure 3). $\hat{T}=$ estimate of the degree of improvement in antidepressant relative to placebo group members at the specified value of Hamilton Rating Scale of Depression (HRSD).

zero, which it was in this case, confirming the prediction given by Kirsch and colleagues (Table 1, Model 1). The slope shows that drugs had increasing success relative to placebo as initial depression increased. Specifically, for every scale value worse on depression that a sample scored at baseline, the drug groups improved by 0.3991 HASD points relative to placebo. Most meta-regression output includes estimates of the slope in two forms (a) the unstandardized form, which we have described; and (b) the standardized form, usually characterized with the Greek symbol, $\beta$, which may be interpreted similarly to a correlation coefficient. In this case, the association between severity and the drug-placebo differences was $\beta=0.52$.

Although in practice many analysts might be satisfied to know the magnitude of the slope and its statistical significance, potentially even more useful is the intercept, which is the value that $\hat{T}$ takes when the moderator variable (or variables) is (are) exactly zero. By default, meta-regression statistical output includes a test of whether the intercep differs significantly from zero, which it was in this case $(T=-7.416 ; 95 \%$ confidence interval $=-11.71 ;-3.12 ;$ Table 1 , Model 1). In other words, we are $95 \%$ confident that the difference in improvement will be in the range from -11.71 to -3.12 for a sample with an initial mean HRSD value of 0 . Thus, if a sample's mean baseline depression is exactly zero, this equation predicts that groups receiving placebo would average 7.416 HRSD points less depression than groups receiving antidepressants. This example helps to illustrate one reason why meta-regression intercepts are so commonly ignored in practice- - because they so often yield unrealistic values. In this particular individ to individual to score 0 on the HRSD, a sample could not have a mean score of exactly 0 unless it also had zero no need for antide

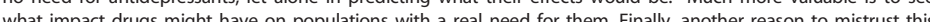
estimate is that there were no observations so low in the database, and therefore, the estimate is a failly extreme extrapolation.

Imagine that we want to know what impact antidepressants should have on patients whose level of depression is $\mathrm{HRSD}=17$, which is the lowest value observed in this particular sample of studies and is conventionally interpreted as a moderate depression. Inserting this value into the equation of the line yields

$$
\hat{T}=-7.416+0.3991(17)=-0.6313 \text {. }
$$

'Of course, if desired, other confidence intervals may be estimated other than the usual default of 95\%; commonly utilized instead are $90 \%$ intervals, which will be more liberal-narrower, and $99 \%$ intervals, which will be more conservative-wider. We return to this issue
in the concluding section, General Limitations and Potential Uses of These Strategies, where we advocate routinely using more conservative estimates than 95\%, particularly under fixed-effects assumptions.
${ }^{2}$ Of course, as Thombs et al. (2011) discussed, it is possible that in reality, an individual who scores 0 on a standardized scale may in fact have elevated levels of depression that are not detected by the scale.
In other words, the equation predicts that members of the drug group will improve less (by 0.6313 HRSD values) than members of the placebo group. Although it is simple to determine a predicted value using th equation of the line, to create a confidence interval around it, one must use the moving constant technique. ${ }^{3}$ Specifically, subtracting 17 from every observation of baseline severity will effectively move the intercept to a value of 17. Now, when we re-run the model, the results show not only the same estimated value of $\hat{T}$ the equation implied, -0.630 but also a confidence interval for it $(-2.04,0.78)$, as Model 2 in Table 1 shows. Thus, for samples with moderate levels of depression, no advantage is likely to be seen for those taking antidepressants (relativ to those taking placebo). Note that the confidence interval around this value is also much narrower (Figure 1 panels b \& c), which makes sense because there are some data available at this point along the moderator variable which to base an estimate (see Figure 1, panel a).

Table 1 uses the same procedure to estimate other confidence intervals across the observed range of the moderator variable. A significant effect of drug is evident when the mean HRSD value reaches 23 (Model 4)

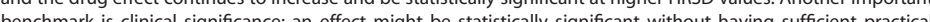
impact in benchmark was achieved somer abserved initiol depression value, 29 (Model 6) the confidence interval no longer includes the value 3 . Thus, on the average for studies whose samples had mean levels of initial depression this high, the observed values exceeded the clinical significance criterion.

For the purpose of illustration, we estimated two more models that move beyond the observed range of the moderator variable. Just as the confidence interval for a sample with zero depression had a wide range, so too does the confidence interval for a sample of extremely depressed patients (46 on the HRSD), in Model 8 . Thes extreme examples might strike some readers as controversial: conventionally, statisticians restrict estimates to observed ranges of moderator variables, but there are times when it is important to project findings beyond what was observed, such as when earth scientists project climate change over centuries. As an example, earth scientists commonly project estimates far into the future, such as how much the earth will warm by the year 2100 and even centuries farther into the future (Solomon et al., 2009), or how high the seas will rise given this amount of warming. Obviously, such predictions can have profound ramifications. Having some confidence in how conditions may change can help community planners protect their territories and maximize outcomes.

The aforementioned models imply how one can estimate confidence bands around the predicted values defined by $\hat{T}$ s. Such is the logic of regression, whether in terms of regular regression with primary-level data e.g., Myers and Well, 2003) or with meta-analytic data (Viechtbauer, 2010b). One can move the intercept across the values of a moderator and plot the estimate and its confidence interval. The problem with moving it in such a coarse fashion is that if the points estimated are very distant from each other, a graph based on this procedure may have poor smoothing between the estimates and might badly estimate certain regions along the moderator variable. Greater accuracy would result if the estimates moved in much finer increments along the moderator variable. In the current example, 1001 i erations that each move the intercept by 0.0123809 of a unit of depression creates an extremely precise estimate of the confidence bands. If we start with mean depression at its minimum observed value, 17.0631, then after 1001 iterations, the intercept would estimate the maximum observed level of mean depression in these samples, 29.444, and the resulting confidence bands will be smoother. Of course, such fine gradations might not be necessary unless a very high resolution graph is needed, such as when it will be printed in a very large size. Our Appendixes list syntax to show how one can create such graphs using two popula statistical platforms, and Figure 1 (panel b) shows such a graph. A significant difference exists when the confidence bands no longer include the red line indicating exactly no difference. A clinically significant difference xists when the confidence bands exceed the green reference line.

As we have illustrated, the moving constant strategy is also helpful for producing point estimates at points of interest, with no graphs at all. To examine estimates along a continuous moderator variable that is examined Ploting estimates at the meanormedian values of then

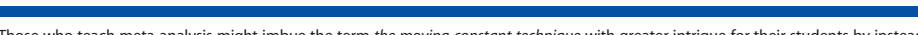

Those who teach meta-analysis might imbue the term the moving constant technique with greater intrigue for their students by instead
calling it the secret of the moving constant. Indeed, it may effectively be a secret to those who have not yet realized its potential. "It is worth noting, as did Kirsch and his colleagues, that statistical inferences associated with this level of depression should be taken with caution, as there was only one case in the sample with an mean depression lower than 23 on the HRSD. It is for this reason that
the confidence interval is relatively wide for estimates of $\mathcal{T}$ with initial mean $\mathrm{HRSD}=17$ (in Table 1 compare Model 2 with Models 4 . sWe searched unsuccessfully for a meta-analysis within the social sciences that had explicitly projected results beyond the limits of moderator. We believe the practice could be quite useful in some circumstances. For example, one might imagine a scenario in which a meta-analysis examines whether a given intervention improves health for a stigmatized group. Prejudice levels by those living in the communities where these trials are done might vary from medium to high. Let us imagine that the meta-analysis shows that the would have if prejudice was low or zero. Such a pattern if confirmed, might suggest that structural interventions to reduce stigm would multiply the impact of the intervention. Hence, using the moving constant technique might be put to fruifful use to projec results into unexplored terrain, which then might stimulate more direct research on the subject. 
may exist as well, such as projecting results beyond the observed range of the moderator variable. Thus, an analyst could plot $\hat{T}$ at specified points along the continuum implied by the moderator variable and report the estimated weighted $\hat{T}$ and the confidence interval around this estimate.

Potential problems with bands produced by graphing functions in conventional software. Because they are easily invoked, one temptation might be to graph such patterns with conventional, widely available software that was written for use with primary-level data and includes ordinary weighted least squares analysis functions. Fo example, one can use SPSS (BBM SPSS Statistics, 2010) to plot confidence bands around weighted regression lines, but because the graphing solution models the standard error of the regression coefficients (including the intercept) differently than conventional meta-analytic statistics in relation to their unstandardized coefficients, $\hat{\beta}_{j}$ the confidence bands and intervals will almost always differ from those implied by the meta-analytic regression model (see Lipsey and Wilson, 2001, pp. 138-140). Hedges and Olkin (1985) noted that "standard errors for $\hat{\beta}$ printed by the program are incorrect by a factor of $\sqrt{M S_{E}}$, where $\mathrm{MS}_{\mathrm{E}}$ is the error or residual mean square for the regression" ( $\mathrm{p}$. 174, italics in original) because standard software does not incorporate the known variance estimate for the meta-analytic data (Konstantopoulos and Hedges, 2004). ${ }^{6}$ Although Hedges and Olkin made their statement in reference to fixed-effects meta-regression models, models that incorporate random-effects assumptions are just as susceptible to the problem. ${ }^{7}$ Future research should address this issue.

To keep graphical depictions of meta-analytic results squarely in accord with the underlying inferential statistics, therefore, analysts are well advised to avoid using standard software for graphing meta-regression results, or at least to double-check that the confidence bands match the meta-analytic model. The logic we outlined in this section can be used for such a check: Estimate the confidence intervals for a value along the moderator dimension in question and compare them with the figure. In Figure 1 (panel c), the dashed lines indicate the confidence bands implied by a meta-regression following mixed-effects assumptions; gray bands resulted by invoking the weighted graphing function in primary-level statistical software (i.e., using the STATA command twoway lifti). These bands (shaded a a) are cleanly wide, thus, statistical and clinical inference will deviate at certain points along the moderator.

Importantly, ordinary weighted least squares analyses do not always overestimate the widths of confidence bands: in fact, the difference can go in either direction. As Hedges and Olkin (1985), earlier, implied, the bias is more conservative (wider confidence intervals and bands) to the extent that $\sqrt{\mathrm{MS}_{\mathrm{E}}}$ exceeds 1 , more libera (narrower intervals and bands) to the extent that $\sqrt{\mathrm{MS}_{\mathrm{E}}}$ is less than 1 , and equivalent when $\sqrt{\mathrm{MS}_{\mathrm{E}}}=1$. Consequently, using ordinary weighted least squares analysis functions bands will be justified only in the very rare instance when $\sqrt{\mathrm{MS}_{\mathrm{E}}}=1$.

The moving constant technique in more complex meta-regression models

Multiple-moderator models. To this point, we have used a bivariate meta-regression example to make use of the intercept completely transparent Now, we turn to a more complicated example that better maps on to the target problem. Assume we have a meta-analysis of studies using exercise to impact quality of life in cancer survivors, such as that Ferrer and colleagues (Ferrer et al. 2010) recently conducted. Imagine that we want to illustrate a model with three moderator variables that we find plausible based on a reading of the literature: (a) the quantity of aerobic metabolic equivalents of task (METs); (b) its quadratic term (i.e., aerobic METs'); and (c) the percentage of the sample that is female. Aerobic METs define how active one is: At rest, one exerts one MET; at six METs, one does vigorous exercise. The studies evaluate the extent to which bouts of exercise that accrue over time relate to quality of life assessed on standard scales.

Just as in ordinary regression, a model with quadratic effects must incorporate the linear effect because otherwise, a statistically significant quadratic effect might in reality be a linear effect. Initially, let us examine trend across the range of aerobic METs, holding the impact of sample gender constant at its sample mean of $79 \%$ female; thus, gender is mean-centered at $79 \%$ in this analysis. Although all-male and all-female samples appeared from following these steps (Appendix I). We see that the impact of exercise is statistically significant across the range from medium (four MET) to very high (eight METs) targeted aerobic activity. No study had a value over

"Similarly, standard meta-regression statistical output under most if not all standard statistical packages, rarely list the MS $\mathrm{S}_{\mathrm{E}}$ associated

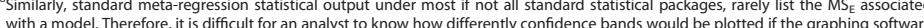
with a model. Therefore, it is difficult tor an analyst to know how differently confidence bands would be plotted it the graphing software
were used. As we mentioned in the text, the moving constant technique can be used to determine whether the bands match metaanalytic assumptions.

7Of note, under heterogeneity, meta-regression models that incorporate random-effects assumptions are certain to have smaller MS
values than would completely fixed-effects versions of these models, and indeed often exhibitit MS , values of about 1 in practice. values than would completely fixed-effects versions of these models, and indeed often exhibit $M S_{E}$ values of about 1 in practice,
Meanwhile, completely fixed-effects versions usually have $M S_{E}$ values much larger than 1 . Thus, if appropriate weights are used (see Appendix I, Step 4), conventional graphing software will often produce reasonably accurate graphs in the case of meta-regressions incorporating random-effects assumptions.
6.25 METs, so after that point, as discussed earlier, any regression line and confidence bands beyond this point amount to a prediction of what would happen at these higher activity points. Figure 2 (panel b) takes it to greater extremes by projecting estimates all the way to aerobic MEIs $=12$, far higher than any study evaluated. Initially, note that although the confidence bands in this graph in the portion of the graph up to METs =8 appear to be narrower, they are in fact the same; it is just that the maximum value of the $y$-axis is now much larger, to $S M D=6$. Nonetheless, one can see that the confidence intervals widen dramatically, just as they should, because there are no obserstions in that region. The figure is also quite implausible from the standpoint that the largest observed efect size is less tha $r$
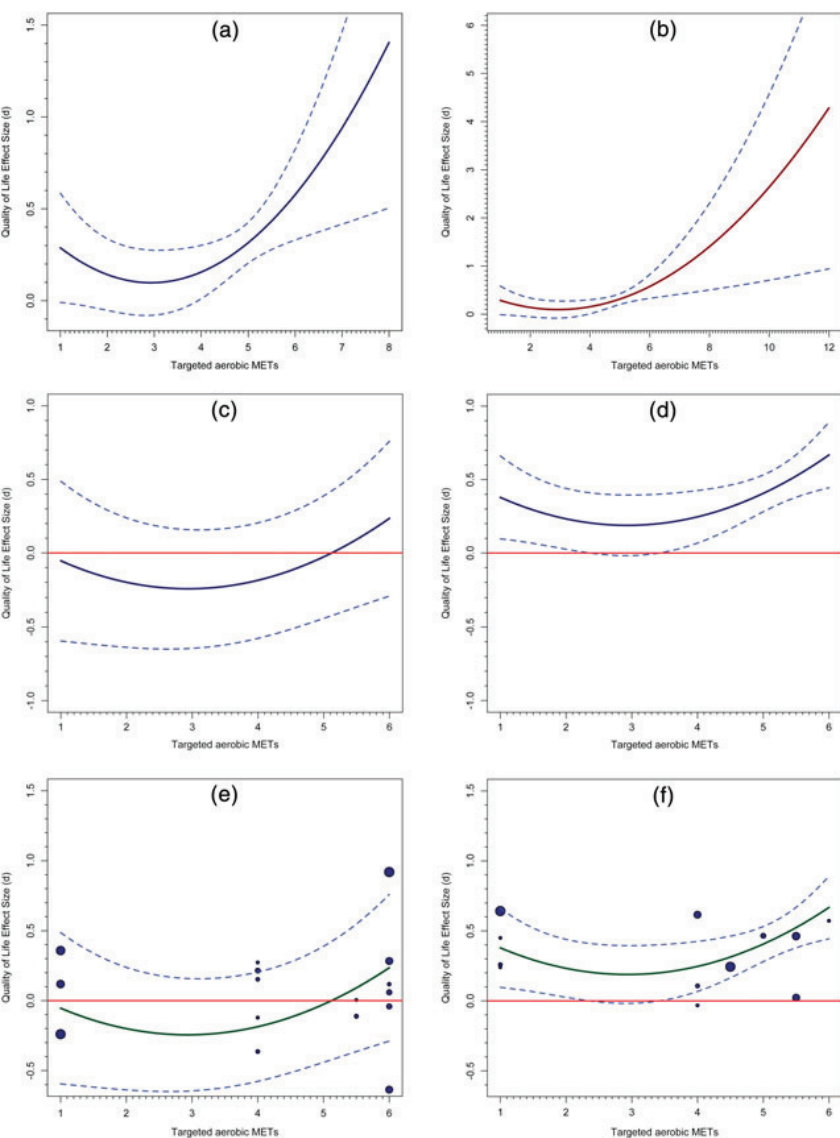
Figure 2. Complex meta-analytic models plotted under differing circumstances implied by the models. Specifically plotted is the relation
between intensity of aerobic exercise on quality of life improvements for cancer survivors (see text for specific descriptions of each panel).
$\mathrm{d}$, standardized mean difference 
undertake such a program. An analyst might use a figure such as this one if he or she was trying to encourage scholars to undertake such trials and to see whether still greater improvements in quality of life can occur. It is important to understand that because gender was zero-centered in these models, strictly speaking, graphs such as those shown in Figure 2 (panels a and b) plot the estimated improvement of various amounts of aerobic exercise, adjusted so that they reflect samples with 79\% females (the mean). Our model showed that samples with larger percentages of females had greater success improving quality of life through exercise. Thus, the plotted line is logically farther from zero for samples that are more female and closer to the line for samples that are more male. The same sequence that produced Figure 2 (panel a) was followed for these two extremes. In two new instantiations, we hold gender constant at $0 \%$ or $100 \%$, respectively. Figure 2 (panels c and d) shows the estimates for samples of men and women, respectively. Although both lines show the curvilinearity implied by the quadratic function, the line is indeed farther from zero for female samples and closer to zero for male samples. Indeed, aerobic exercise would appear to have little impact on quality of life for samples of men unless more intense appers to improve quality of life on the average but especilly at higher intensities. Note to that the confidence appass are narrower for female than for male samples, consistent with the fact that there were more observations for the former group. As with any meta-analytic model the analyst should take care to note such limitations when interpreting such findings. The authors of this particular meta an sysis used exactly these procedures to evaluate interpreng such models of the relation, showing that the quadratic function did not a procedures to evaluate exercised steadily over a few months; interested readers should consult their article (Ferrer et al. 2010), which lists other limitations of this particular meta-analysis.

Complications involving categorical variables. Up until this point, our examples of used continuous variables as moderators. Note that the moving constant technique can help an analyst avoid the problem of bifurcating continuous variables; that is, the models themselves can produce estimates for particular levels of the continuous varlables, obviating the need to artificially dichotomize them. Yet, categorical variables are commonly incorporated in meta-analytic research and they present some special complications. Imagine we are still pursuing the same meta-analytic model as in the preceding paragraphs, but that now gender is represented as two categories, male and female, instead of as a continuous variable, percentage of females in the sample. Orthogona coding gender so that male samples take -1 and female samples take +1 effectively controls the gender effec when estimating other moderators' effects. Now, interpretation proceeds as we have already described.

Imagine further what the results would imply if one instead dummy-coded the sample gender variable so that male samples tak os and female samplestake 1 , leaving aerobic metric. Now, the intercpt has little or no meaning because the MET scal begins not at value 0 but at 1 , which implies be the rest. The solution of course is to commence with the intercept of our model reflecting the lowest value of the scale (1) rather than the meaningless value of 0 . Finally, complexities in such models increase as the numbers of moderator variables increase, but the underlying logic to display the results is the same as we have presented here For erample, Johnson and colloages (Jhnson et al 2003) fit a meta-regression model with five moderaters in their meta-analysis of studies evaluating human immunodeficiency virus prevention interventions for adolescents. Three variables were continuous and two were categorical (dummy or contrast coded). It took them 14 meta-regression runs to produce estimates for the extremes of each moderator while statistically holding the other moderators' influences constant.

\section{Is zero-centering or contrast-coding moderators always the best solution?}

Some readers may take the preceding examples to imply that one must zero-center or contrast code all moderators except for the one that is the focus of the moving constant technique to portray estimates and their confidence intervals or bands across that moderator. Yet, especially when interactions between moderators exist, it may be preferable to produce estimates that follow differing assumptions. Imagine that, as is often the case, the studies in a particular meta-analysis vary in methodological quality. Suppose we are examining the effects of exercise on depression levels in cancer survivors and expect to see a dose-response curve such that depression improves with greater amounts of exercise. An analyst could regress Ts on exercise dose, study quality, and the interaction of these terms. (Note that the analysis to examine the statistical significance of these terms will likely be more stable if the dose and quality terms are zero-centered before multiplying them to create the interaction term.) Suppose further that the interaction is statistically significant. In such a circumstance, an analyst might well show the dose-response pattern with study quality held constant at a high level because, logically, these are the studies whose results are the most trusted. Alternatively, one might show the dose-response function for both high-quality and low-quality studies. Brown and colleagues (Brown et al., 2011, Table 4) followed just these procedures in their meta-analysis examining the effects of resistance
dose-response pattern was most marked for the highest quality studies.
Caveats about clinical significance

Because no standard for clinical significance yet exists for quality of life outcomes, we have focused instead on statistical significance in this section. That there are many different scales to assess quality of life makes achieving clinical significance standard more difficult but not impossible. Indeed, if one wished to parallel the NICE criterion for depression change, then one could generalize from the fact that their clinical significance criterion specifies the target of a "medium" effect size of SMD $=0.50$, as per the standards given by Cohen (1988). Consequently, a medium effect size implies a change that is visible to the naked eye, with no need for statistics (Johnson and
Kirsch, 2008). The graphs in Figure 2 might be interpreted as failing to meet this clinical significance standard, as none of the confidence bands exceed this value.

\section{General limitations and potential uses of these strategies}

It is worth discussing some other potential uses of the strategies that we have described as well as some genera

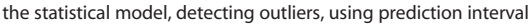

\section{Assumptions underlying the model}

Sources on meta-analysis routinely state how, under heterogeneity, fixed-effects assumptions typically produce overly narrow confidence intervals relative to random-effects and mixed-effects assumptions. To illustrate, Figure 3 portrays the same antidepressant data that appeared in Figure 1. Figure 3 (panel a) follows fixed-effects assumptions, and Figure 3 (panel b) follows random-effects assumptions. Because heterogeneity is present, in a formal sense, the fixed-effects version is incorrectly specified; consequently, it has overly liberal, narrowe confidence bands (Figure 3, panel a) and the random-effects version with its wider confidence bands might instead be favored (Figure 3, panel b). In this case, statistical inference does not dramatically differ between the subject to any number of problems, including non-normal sampling distributions, invalidity, unreliability, and restriction of range, to name only a few (Hunter and Schmidt, 2004; Cohen et al., 2004).

\section{Outlier detection}

Graphs such as we have produced can be helpful in diagnosing problems in the literature; we implied just this unction at the outset, in discussing bivariate meta-regressions. The same holds for the more complex examples we presented in the preceding section. It is most valuable to use the more complex patterns implied by the model; thus, we can understand the in lt is me of some particular relevant moderators while controlling for the effect of others. Figure 2 (panels $e$ and $)$ uses the regression lines and confidence bands from panels $c$ and $d$ overall model, epo-centering gender, would be prone to error becaus it does not account for the gender effect. If we still did so, then relatively large effect sizes would be more likely to be from studies that focused on women and relatively small (or negative) effect sizes would be more likely to have focused on men. That sample gender is because we had to pick which effect sizes to plot in each graph. It seems reasonable to plot cases with 50\% or more men in the former plot and those with $50 \%$ or more women in the latter plot One can see that the regression lines stay closer to studies with larger weights, which illustrates meta-analytic weighting in action. These plots also visually show residuals, which is the difference between the estimated value and the observed value for each case. Those farther from their lines have larger residuals. There are indeed far fewer cases for male cancer survivors, so statistical inferences are quite strained here. Panels e and $f$ both show that there are few studies in the range under METs $=4$, again implying that predictions here are a projection. The quadratic function appears most justifiable for the female sample studies, although there are some outliers that would be worth inspecting. Alternatively, other moderators might improve prediction and eliminate these large residuals.

\section{Prediction intervals}

This article focused most on estimating Ts at certain points along a moderator dimension or dimensions, whic boils down to estimating where the weighted mean $T$ lies along a moderator or set of moderators. Another alternative is to portray the prediction interval in which future studes are likely to be observed in relation to a this strategy addresses the dispersion of effect sizes that one is likely to see in a particular literature. Appendix 

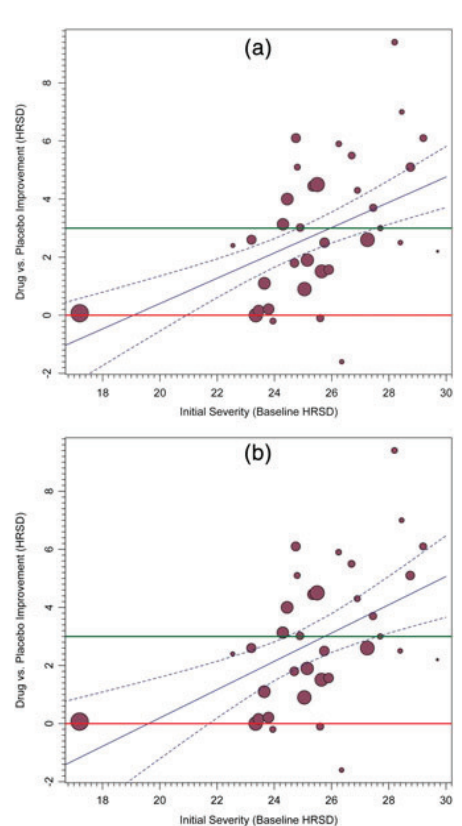

Figure 3. Improvement on depressive symptoms as a function of baseline severity of depression, with each point sized proportionally to its weight in the analysis. (panel a) Moderation pattem showing regression line and $95 \%$ confidence band under fixed -effects assumptions. (panel b) Same as panel a
but under mixed-effects assumptions (iie, with a random-effects constant and a fixed-effects slope). HRSD, Hamilton Rating Scale of Depression

lists steps that can be used in one statistical platform to produce such estimates in tandem with meta-regression models. Figure 4 (panel a) shows a graph with such estimates for the Kirsch antidepressants data, showing that the prediction interval is much wider than the confidence bands. An analyst might display such a graph to develop expectations for how large $T$ will be in a new study that matches a particular level of a moderator variable or variables, making it an adjunct to power analysis strategies.

\section{Bayesian estimates}

The solutions that we described in the preceding two sections are decidedly frequentist in orientation, a practice often labeled classical meta-analysis. Nonetheless, those who wish to construct similar graphs following Bayesian assumptions can do so. In Figure 4 (panel b), we portray the empirical Bayesian estimates of the true effects for each study (once again in the Kirsch antidepressants database), assuming the fitted model is correct (Appendix I). In this graph, the observed SMDs appear as bubbles and the adjusted, true SMDs appear as filled bubbles. Arrows show how the observed SMDs converge on the regression line of the meta-regression model in the empirical Bayesian estimates of the true SMDs. Those cases that

\section{Simultaneous confidence bands}

The strategy that we have emphasized in this article is the pointwise method of constructing confidence bands and intervals. The simultaneous confidence band strategy attempts to control for the expanded error rate that accrues from the evaluating a statistical hypothesis across the range of a moderator variable or variables (Liu et al., 2008; Macskassy \& Provost, 2004; Seber and Lee, 2003; Working and Hotelling, 1929). The pointwise strategy
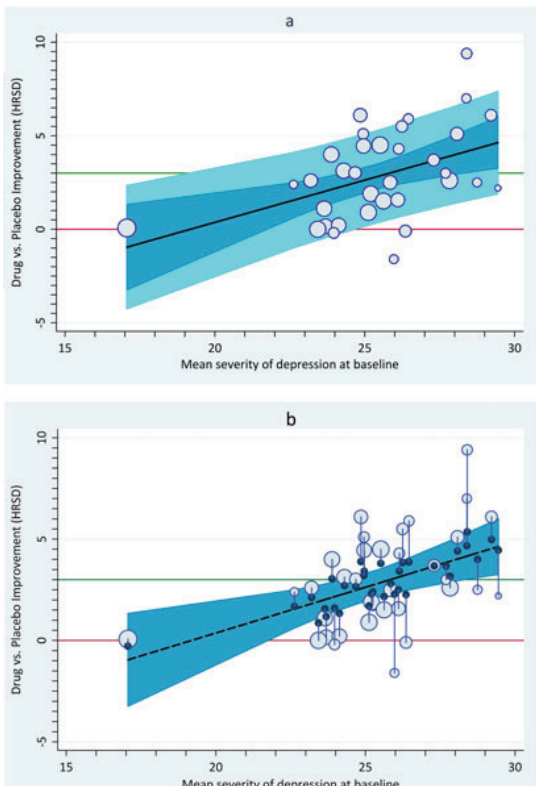
Figure 4. Improvement on depressive symptoms as a function of baseline severity of depression, with each point sized proportionally to its weight
in the analysis, and following mixede-effects assumptions. (panel a) Moderation pattern showing $95 \%$ prediction intervals depicted in light blue.
(panel b) Observed (open circles) and empirical Bayesian estimates (filled circles) of true effect sizes. HRSD, Hamilton Rating Scale of Depression

we have illustrated in this article would presumably suffer from heightened Type I error rates, stemming from examining whether $\hat{T}$ is significant at different levels of a moderator variable or variables. Note that in theory, a moderator dimension can be divided into infinite increments and heightening Type I $(\alpha)$ error rates. The Bonferron correction is often applied to reduce Type lerror rates by dividing a by the number of tests being evaluated but cannot

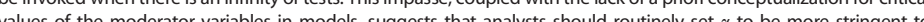
values of the moderator variables in models, suggests that analysts should routhely set $\alpha$ to be more stringent to compensate or else interpret the is to be encouraged As we have stated, these models are already more conservative than fully fixed effects versions, under heterogenety. Impotenty to de, there appears to be no eaily avilable stategy for applying the

\section{Practical limitations}

It is worth discussing when graphs such as we have produced here might be most valuable. Given that most metaanalyses investigate numerous moderator variables, plotting all patterns might often prove impractical. If the gool is comprehensiveness, then tables of output are much more compact than numerous figures. Instead, the pattern that deserve the most practical attention are the ones that analysts should graph-the ones that they want to help tell the story of their meta-analytic results. Finally, note that, in practice, successful graphing is often an intensive, iterative practice in which the analyst produces successive versions of the same pattern until the right combination of informativeness and aesthetic value is achieved (Tufte, 2001).

One potential limitation of the steps we have listed here to depict metaregession results is financial. True,

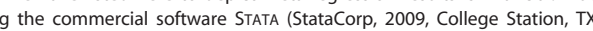
(Appendix I). The macros freely given by Lipsey and Wilson (2001) also are available for sPSS and SAS in addition 
to Stata, but these statistical platforms are all commercial. ${ }^{8}$ Those who are students rather than professionals often can get discounted prices on such software. Fortunately, all or nearly all of the solutions we suggest are also available in the open-access statistical software R (see Burns, 2006), for which Viechtbauer (Viechtbauer, 2010a; Viechtbauer, 2010b) has written a sophisticated suite of meta-analysis protocols, metafor. Appendix II shows how we used this software to create graphs such as in Figure 2 and Figure 3.

\section{Conclusion}

Several prominent sources have given careful attention to graphing primary-level data and offer sage advice that meta-analysts might well also incorporate. Specifically, Tufte (Tufte, 2001; Tufte, 2006) argued for more carefu integration of graphical and textual information, much in the way in which Leonardo da Vinci's journals richly illustrate. Research has shown that the modern practice of separating text and graphics yields poore communication of the targeted scientific information (Sweller et al., 1990). Two other sources provide very helpfu advice about how best to represent more nuanced aspects of scientific databases (Cleveland, 1984; Lane and Sándor, 2009). To date, such sources emphasize primary-level investigations almost entirely to the exclusion of meta-analysis. The current article has emphasized how many of these principles of effective graphical display generalize particularly well to effect size information.

In sum, the strategies we have described here should enable meta-analysts to create accurate graphs of metaregression results and more effectively communicate scientific information. Because graphical depictions of as we have detailed should be an indispensible tool in meta-analysts' toolkit.

\section{Appendix I: Calculating and displaying confidence intervals and bands using \\ meta-regression in STATA}

(1) First make sure that STATA has installed the most recent version of the metareg command by using the "lookup" command:

lookup metareg

\section{Keyword search}

Keywords: metareg

Search: (1) Official help files, FAQs, Examples, SJs, and STBS

Search of official help files, FAQs, Examples, SJs, and STBs

SJ-8-4 sbe23_1 ............... Meta-regression in Stata
(help metareg if installed) .... R. M. Harbord and J. P. T. Higgins

Q4/08 SJ 8(4):493-519

presents a revised version of the metareg command, which

performs meta-analysis regression on study-level summary

data

Selecting the sbe23_1 entry (above) lets a user install or update to incorporate the metareg command presented by Harbord, which was first published in 2008 (Harbord and Higgins, 2008), updated from an earlier version that STATA published in 1998. If the metareg macro given by Lipsey and Wilson (2001) has previously been installed, then it will need to be renamed in order for the alternative to operate. For the graphs we produced for this paper, we used STATA version 11.2. After installing or updating the command, it can be used to estimate meta-analysis

(2) After installing or updating the command, it can be used to estimate meta-analysis regression (viz. metaregression) models. The basic form of the command follows this format:

. metareg T moderator1 moderator2, wsse(se_T)

where $T$ is the effect size, moderator 1 and moderator2 are moderator dimensions, and se_T is the standard error where $T$ is the effect size, modem "mixed-" versus "random-effects" assumptions). If inverse variance wights, TW, have bsion calculed

${ }^{8}$ Readers can request from the authors a version of the current strategies for constructing confidence bands using the macros by Lipsey Readers can request for
and Wilson (2001).
$T$ (see, e.g., Lipsey and Wilson, 2001, Table 3.2) but not se_T, then to use the metareg command, it is first necessary to calculate se_T:

gen se_ $T=1 /$ sqrt(TW)

For example, in the antidepressants data described in the main text, the command

metareg diffimp meanbase, wsse(se_diffimp) graph

where diffimp is the $T$ (difference in improvement in depression between drug and placebo groups), meanbase is the mean severity of depression at baseline, and se_diffimp is the standard error for each $T$, produces statistica output for the meta-regression model and a so-called bubble graph, where the bubbles are individual Ts sized

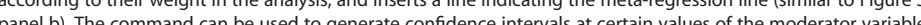
such as the lowest and highest observed values. For example,

.gen tempvar $=$ meanbase- 17.063

where 17.0631 was the lowest observed mean severity of depression at baseline, and tempvar is intended to replace meanbase in the re-instantiated command:

metareg diffimp tempvar, wsse(se_diffimp) graph

which moves the constant or intercept to the value of 17 (the graph now literally shows the intercept at the zero point of the newly calculated variable, tempvar, on the left side of the graph). To obtain an estimate at the highest observed mean value, 29.444:

replace tempvar $=$ meanbase- 29.444

and we re-use the tempvar variable and re-instantiate the metareg command:

metareg diffimp tempvar, wsse(se_diffimp) graph

and the zero point now appears on the right side of the graph. (Note that the graph command will not work with more than one variable.) These models provide confidence intervals for these point estimates but not confidence bands and other additions; for these, see the next steps.

(3) Because the metareg command can be used with post-estimation commands, a number of other possibilities for graphing become possible. Specifically, after running the metareg command, run these commands:

(4) Now the variables saved in step 3 can be used for plotting. To produce a graph similar to that in Figure 1 (panel b) . twoway rarea confl confu meanbase $\|$ line fit meanbase $\|$

\begin{tabular}{|c|c|}
\hline Command & Explanation \\
\hline . predict & Saves \\
\hline - predict stdp, stdp & Saves the stand \\
\hline - predict stdf, stdf & Saves the standard error of the forecast in a variable called stdf \\
\hline . predict xbu & $\begin{array}{l}\text { Saves empirical Bayes estimates (predictions including random effects) } \\
\text { in a variable called } x b u\end{array}$ \\
\hline $\begin{array}{l}. \text { local } t=\text { invttail }(e \\
\left.\left(d_{f} r\right)-1,0.025\right)\end{array}$ & $\begin{array}{l}t \text { is used in calculating } 95 \% \text { confidence and prediction bands; for } 90 \% \text {, } \\
\text { replace } 0.025 \text { with } 0.05 \text {; for } 99 \% \text { replace it with } 0.005\end{array}$ \\
\hline . gen $\operatorname{confl}=$ fit & $\begin{array}{l}\text { Calculate lower confidence band (using standard error of the prediction), } \\
\text { confl }\end{array}$ \\
\hline - gen confu $=$ fit $+t^{\prime} t^{*}$ stdp & $\begin{array}{l}\text { Calculate upper confidence band (using standard error of the prediction), } \\
\text { confu }\end{array}$ \\
\hline - gen predl $=$ fit $-{ }^{\prime} t{ }^{\prime}{ }^{\text {st }}$ & $\begin{array}{l}\text { Calculate lower prediction band (using standard error of the forecast), } \\
\text { predl }\end{array}$ \\
\hline - gen predu $=$ fit + & $\begin{array}{l}\text { Calculate upper prediction band (using standard error of the forecast), } \\
\text { predu }\end{array}$ \\
\hline
\end{tabular}

where rarea calls an area plot to depict the confidence bands and line plots the predicted values. Note that we tick marks). To produce a bubble graph similar to Figure 3 (panel b): 
.twoway rarea confl confu meanbase $\|$ line fit meanbase $\|$ scatter diffimp meanbase [aw $=1 /$ se_diffimp^2], msymbol(Oh) \|

This command sizes bubbles according to the fixed-effects variance estimate; the random-effects equivalent can be produced by summing the random-effects variance component, $\tau^{2}$, and the fixed-effects variance: gen $T W r=1 /\left(\right.$ fevar $\left.+T^{2}\right)$

where fevar is $1 / T W$ and $\tau^{2}$ is a constant value taken from the statistical output. Now, the equivalent command sizes the bubbles according to their total weight:

twoway rarea confl confu meanbase || line fit meanbase $\|$ scatter diffimp meanbase [aw = TWr], msymbol(Oh)

Note that just like the variables in step 3, TWr would need to be recalculated for each such model because $\tau^{2}$ and the other variables vary from model to model.

(5) To show the prediction interval surrounding the meta-regression line, such as in Figure 4 (panel a): . twoway rarea predl predu meanbase || line fit meanbase \|

(6) To display the empirical Bayesian estimates along with the meta-regression line and confidence bands, . twoway || rarea confl confu meanbase || line fit meanbase || scatter xbu meanbase, msymbol(t) ||

Or, to overlay the observed Ts along with their predicted true values assuming the fitted model is correct along with the confidence bands, similar to Figure 3 (panel d):

twoway \| rarea confl confu meanbase || line fit meanbase || scatter diffimp meanbase [aw = TWr], msymbol (Oh) || scatter xbu meanbase, msymbol(t) ||

\section{Appendix II: Calculating and displaying confidence bands in meta-regression}

\section{using metafor with $\mathrm{R}$}

We used commands similar to these using the R statistical package in order to create the graphs in Figures 2 and 3 .

(1) Initially, install $R$ and the package metafor, which is available via the Comprehensive R Archive Network (CRAN) at http://CRAN.R-project.org/package=metafor, the author's website at http://www.wvbauer.com/, or through a direct command within R: install.packages("metafor") (one needs an internet connection and appropriate access rights on the computer). Then, open metafor and load the data set, which in this

library("metafor")

data("QoL", package="metafor")

data( QoL, package="metafor)

(2) Now, fit the meta-analytic model with the quadratic effect of targeted aerobic METs and proportion of women using the command rma.uni( ) as follows:

aeromet $<-$ rma(d, vi, mods $=\sim$ aerobicmet + I(aerobicmet $\left.{ }^{2} 2\right)+$ expwomen, subset $=(($ design $==1$ |design == 0) \&amp; fup == 2), data $=$ QoL, method="ME")

The model has three independent variables: aerobicmet, aerobicmet ${ }^{2}$ (the two aerobic METs terms), and expwomen (percentage of women in the sample). If fixed-effects assumptions are desired instead of mixed-effects, then replace $\mathrm{ME}$ with $\mathrm{FE}$. The "subset=" portio
cases that we wished to model.

(3) To obtain the predicted values:

predsaeromet $<$ - predict(aeromet, newmods $=\operatorname{cbind}(\operatorname{seq}(1,8,1), \operatorname{seq}(1,8,1) \wedge 2,79))$

wi $<-1 /$ sqrt(QoL\$vi)
size $<-0.5+3 *(w i-\min (w i)) /(\max (w i)-\min (w i))$

The value 79 is the sample mean for percentage of women.
(4) Then, plot the predicted values and the confidence bands:

plot(QoL\$aerobicmet, QoL\$d,type="n",xlab = "Targeted aerobic METs", ylab = "QoL Effect Size (d)", $x \lim =c(1,8)$, ylim=c(-0.1, 1.5

lines(seq $(1,8,1)$, predsaeromet $\$$ pred, col = "navy")

(l)

lines $($ seq $(1,8,1)$, predsaeromet 5 ci.ub, Ity = "dashed", col=" blue")

(5) To plot the estimates beyond the observed METS range of the studies (in this case up to 12 , such as in Figure 2 panel b):

$$
\begin{aligned}
& \begin{array}{l}
\text { predsaeromet }<- \text { p } \\
\text { wi }<-1 / \text { sqrt }(\mathrm{Q} L \mathbf{L} \text { V } \\
\text { size }<-0.5+3 *(\text { wi }
\end{array} \\
& \text { size }<-0.5+3 *(\text { wi }-\min (w i)) /(\max (w i)-\min (w i)) \\
& \text { plot(QoL\$aerobicmet,QoL\$d,type="n",xlab = "Targeted aerobic METs", } \\
& \text { ylab = "QoL Effect Size (d)", } x \lim =c(1,12), y \text { lim }=c(-0.1,6)) \\
& \text { lines(seq }(1,12,1) \text {, predsaerometspred, col ="red") } \\
& \text { lines }(\text { seq( }(1,12,1) \text {, predsaerometșci.lb, Ity = "dashed", col=" blue") } \\
& \text { lines(seq }(1,12,1) \text {, predsaeromet\$̦ci.ub, Ity = "dashed", col=" blue") }
\end{aligned}
$$

(6) To plot the observed values for a particular subset of the sample, initially, create a subsample: dwomen $<$ - subset $(Q \circ L$, expwomen $==100 \&($ design $==1 \mid$ design $==0) \&$ fup $==2$ dmen <- subset( $(\mathrm{L} L$, expwomen $==0$ )

(7) Then plot studies with, for example, primarily men initially (as in Figure 2, panel c):

$$
\begin{aligned}
& \text { plot(dmen\$aerobicmet,dmen\$d, pch = } 21, \text { col = "black", bg = "navy", cex = size, } \\
& \text { xlab = "Targeted aerobic METs", ylab = "QoL", ylim=c(-0.6, 1.5), xlim=c(1, 6)) } \\
& \text { lines(seq(1,6,1), predsaeromet\$pred, col="dark green") } \\
& \text { lines(seq(1,6,1), predsaeromet\$ci.l., Ity = "dashed", col=" blue") } \\
& \text { lines(seq(1,6,1), predsaeromet\$ci.ub, Ity = "dashed", col=" blue") } \\
& \text { abline(h = 0, Ity = "dotted", col="red") }
\end{aligned}
$$

\section{Acknowledgements}

We express our appreciation to Estrellita Ballester, Justin C. Brown, Dipek Dey, Adam R. Hafdahl, Larry V. Hedges, David Hoaglin, Jessica M. LaCroix, Mark W. Lipsey, Tom Stanley, Michelle R. Warren, and three anonymous reviewers for helpful comments on previous drafts of this article, or on a related presentation at the 5th Annua Meeting of the Society for Research Synthesis Methodology (Ottawa, Ontario, Canada), or both. We also thank 2002 that utim MH58563 and K18-A1094581 to Blair T. Johnson.

\section{References}

Anzures-Cabrera, J, Higgins JPT. 2010. Graphical displays for meta-analysis: An overview with suggestions for practice. Research Synthesis Methods 1: 68-80.

Berkey, C, Hoaglin DC, Mosteller F, Colditz GA. 1995. A random-effects regression model for Meta-analysis. Statistic in Medicine 14: 395-411.

Borenstein, M, Hedges LV, Higgins JPT, Rothstein HR. 2009. Introduction to Meta-Analysis. John Wiley \& Sons, Wes Sussex.

Borman, G, Grigg JA. 2009. Visual and narrative interpretation, in The Handbook of Research Synthesis and Metaanalysis. 2nd Ed., (Eds: Cooper H, Hedges LV, Valentine JC) Russell Sage Foundation, New York, NY 497-519.

Brown, J, Huedo-Medina TB, Pescatello LS, Pescatello SM, Ferrer RA, Johnson BT. 2011. Efficacy of exercis interventions in modulating cancer-related fatigue among adult cancer survivors: a meta-analysis. Cancer Epidemiology Biomarkers and Prevention 20: 123-133.

Burns, P. 2006. R relative to statistical packages: a look at STATA, SAS and SPSS. Accessed 10 June 2010 (http://lib.stat. cmu.edu/S/Spoetry/Tutor/R_relative_statpack.pdf). 
Cleveland, W. 1984. Graphs in scientific publications. The American Statistician 38: 261-269.

Cohen, J. 1988. Statistical Power Analysis for the Behavioral Sciences. 2nd Ed. Lawrence Erlbaum Associates: Mahwah Hillsdale, NJ.

Cohen, J, Cohen P, West SG, Aiken LS. 2003. Applied multiple regression/correlation analysis for the behavioral sciences. 3rd Ed. Lawrence Erlbaum Associates Publishers: Mahwah, NJ.

Cooper, H, Hedges LV, Valentine JC. 2009. The Handbook of Research Synthesis and Meta-analysis. 2nd Ed. Russell Sage Foundation, New York, NY.

Ferrer, R, Huedo-Medina TB, Johnson BT, Stacey R, Pescatello L. 2010. Exercise interventions for cancer survivors: a meta-analysis of quality of life outcomes. Annals of Behavioral Medicine 41: 32-47.

Hayter, A, Liu W, Wynn HP. 2007. Easy-to-construct confidence bands for comparing two simple linear regression lines. Journal of Statistical Planning and Infererence 137: 1213-1225.

Hedges, L. 1992. Meta-analysis. Journal of Educational Statistics 17: 279-296.

Hedges, L, Olkin I. 1985. Statistical Methods for Meta-analysis. Academic Press, Orlando, FL.

Hedges, L, Vevea JL. 1998. Fixed- and random-effects models in meta-analysis. Psychological Methods 3: 486-504. Higgins, J, Green S. 2009. Cochrane handbook for systematic reviews of interventions, version 5.0.2. Accessed 18 February 2011 (www.cochrane-handbook.org).

Higgins, J, Thompson SG. 2002. Ouantifying heterogeneity in a meta-analysis. Statistics in Medicine 21: 1539-58. Huedo-Medina, T. Johnson BT. 2010. Modelos Estadísticos en Meta-Análisis [Statistical Models in Meta-Analysis]. Netbiblio, La Coruña.

Hunter, J, Schmidt FL. 2004. Methods of Meta-Analysis: Correcting Error and Bias in Research Findings, 2nd Ed. Sage Publications, Thousand Oaks, CA.

IBM. SPSS Statistics. 2010. Release 19.0, SPSS Inc., Chicago, IL.

Johnson, BT, Carey MP, Marsh KL, Levin KD, Scott-Sheldon LAJ. 2003. Interventions to reduce sexual risk for the human immunodeficiency virus in adolescents, 1985-2000. Archives of Pediatrics and Adolescent Medicine 157: $381-388$.

Johnson, BT, Kirsch I. 2008. Do antidepressants work? Statistical significance versus clinical benefits. Significance 5 54-58.

Kirsch, I, Deacon BJ, Huedo-Medina TB, Scoboria A, Moore TJ, Johnson BT. 2008. Initial severity and antidepressant benefits: a meta-analysis of data submitted to the Food and Drug Administration. PLoS Medicine $\mathbf{5}$ (e45): 0260-0268.

Knapp, G, Hartun J. 2003. Improved tests for a random-effects meta-regression with a single covariate. Statistics in Medicine 22: 2693-2710.

Konstantopoulos S, Hedges LV. 2004. Meta-analysis, in The Handbook of Quantitative Methods in the Social Sciences, (Ed: Kaplan M), Sage Publications, Thousand Oaks, CA 281-297.

onstantopoulos, S, Hedges LV. 2009. Analyzing effect sizes: fixed-effects models, in The Handbook of Research Synthesis and Meta-Analysis. 2nd Ed., (Eds: Cooper H, Hedges LV, Valentine JC).Russell Sage Foundation, New York, NY 279-294.

Lane, D, Sándor A. 2009. Designing better graphs by including distributional information and integrating words, numbers, and images. Psychological Methods 14: 239-257.

N, Schmid CH. Olkin I. 2006. The case of the misleading funnel plot. British Medical

Light, E, Singer JD, Willett JB. 1994. The visual presentation and interpretation of meta-analyses, in The Handbook of Research Synthesis and Meta-analysis, 2nd Ed., (Eds: Cooper H, Hedges LV, Valentine JC) Russell Sage .

1. Practical Meta-Analysis. Sage Publications, Thousand Oaks, CA.

Liu, W, Lin S, Piegorsch WW. 2008. Construction of exact simultaneous confidence bands for a simple linear

regression model. International Statistical Review 76: $39-57$.
Macskassy, S, Provost, F. 2004. Confidence Bands for ROC Curves: Methods and an Empirical Study. Proceedings of the First Workshop on ROC Analysis in Al.

Myers, J, Well AD. 2003. Research Design and Statistical Analysis. Lawrence Erlbaum Associates, Mahwah, NJ.

Raudenbush, S. 2009. Analyzing effect sizes: random-effects models, in The Handbook of Research Synthesis and Meta-Analysis, 2nd Ed., (Eds: Cooper H, Hedges LV, Valentine JC), 295-315. Russell Sage Foundation, New York, NY. Seber, G, Lee AJ. 2003. Linear Regression Analysis. 2nd Ed. John Wiley \& Sons, Hoboken, NJ.

Solomon, S, Plattner G, Knutti R, Friedlingstein P. 2009. PNAS: irreversible climate change due to carbon dioxide emissions. Proceedings of the National Academy of Science of the United States of America 106: 1704-1709.

StataCorp. 2009. STATA statistical software: release 11, StataCorp LP, College Station.

Sweller, J, Chandler P, Tierney P, Cooper M. 1990. Cognitive load as a factor in the structure of technical material. Journal of Experimental Psychology 119: 176-192.

Thombs, B, Coyne JC, Cuijpers P, de Jonge P, Gilbody S, loannidis JP, Johnson BT, Patten SB, Turner EH, Ziegelstein AC. 2011. Rething screening for depression in primary care. Canadian Medical Association Journal. DOl: cmaj.111035.
Tufte E. 2006. Beautiful Evidence. Graphic Press, Cheshire, CT.

Tufte, E. 2001. The Visual Display of Quantitative Information. 2nd Ed. Graphic Press, Cheshire.

Viechtbauer, W. 2010a. The metafor package: a meta-analysis package for R. Accessed 10 June 2010 (http $/ / / \mathrm{ww} w$ wvbauer.com/downloads.html).

Viechtbauer, W. 2010b. Conducting meta-analyses in R with the metafor package. Journal of Statistic Software 36 $1-48$.

1. American Statistical Association 24: 73-85. 